\title{
New Signaling approach Improving the VoIP Quality of Service in Ad hoc Network
}

\author{
Salma Rattal \\ EEA\&TI Laboratory \\ Faculty of sciences and \\ techniques \\ Hassan 2nd University \\ Mohammedia-Casablanca \\ Morocco
}

\author{
Abdelmajid Badri \\ EEA\&TI Laboratory \\ Faculty of sciences and \\ techniques \\ Hassan 2nd University \\ Mohammedia-Casablanca \\ Morocco
}

\author{
Mohammed Moughit \\ National school of applied \\ sciences \\ Hassan 1st University \\ Khouribga, Morocco
}

\begin{abstract}
Mobile Ad Hoc NETworks (MANET) are autonomous networks composed of two or more mobile nodes with wireless communication capabilities, but they have no centralized infrastructure network.

VoIP is a technology that allows you to make voice calls using an Internet connection instead of a regular (or analogy) phone line. The quality of service on the VoIP network is spread over several parameters (jitter, MOS, end to end delay, and packet loss rate) which must be taken into account and studied properly during a simulation of a network adopting this technology, which is sensitive to disturbance and dynamic topologies such as ad hoc networks.
\end{abstract}

For this purpose, several studies have been conducted to improve the quality of voice conveyed via an ad hoc network known for its limited resources and constraints of a higher level.

This paper presents a new approach to H.323 signalling in an ad hoc network implementing three Gatekeepers at a time, or the user calculates the distances separating the three gatekeepers to decide to connect to the appropriate Gatekeeper which is the closest geographically.

This new approach is simulated on Opnet Modeler that will calculate the parameters of the obtained QoS and thus can compare them with the parameters of an H.323 signalling system based on a single Gatekeeper.

\section{Keywords}

VoIP, H.323, Ad Hoc, mobility, location.

\section{INTRODUCTION}

An ad hoc wireless network [3] is a collection of mobile/semimobile nodes with no pre-established infrastructure, forming a temporary network. Each node has a wireless interface and communicates with other nodes over a radio or infrared link.

Laptops and PDAs that communicate directly with each other are just some of the nodes of an ad-hoc network. These nodes are often mobile. However, they may also be fixed nodes or Semi mobile nodes.

\section{BIBLIOGRAPHY}

\subsection{Characteristics of ad hoc networks}

Ad hoc networks are often characterized by a dynamic topology due to the fact that nodes change their physical location while moving. This promotes dynamic routing protocols that discover the optimal routes via conventional routing algorithms like distance vector and link state [4]. Another feature is that a host / node has a processor capacity, storage capacity, battery power and very limited bandwidth, it is also called "thin client". This means that the energy consumption must be restricted, thus leading to a limited range of transmission. The radio environment also has special characteristics that must be considered when designing protocols for ad hoc networks.

Multi-hop in a radio environment can lead to improvements in power and transmission capacity. By using multi-hop, nodes can transmit packets with a lower power output.

\subsection{H.323 signalling}

The H.323 is an ITU-T standard [5] for multimedia communications over local area networks that do not guarantee QoS. It addresses call control, multimedia management, bandwidth management and interfaces between LANs and other networks. The entities in the H.323 topology are the endpoints (also called user terminals), gateways, multipoint control units and gatekeeper (GK). The H.323 standard defines procedures for user registration with a gatekeeper, call control and logical channel capabilities negotiation between two or more parties. Usually, in real-time communication between two endpoints, the two endpoints are H.323 terminals. But, in general, the communication could be in a different domain - PSTN or SIP. The H.323 call model is between two H.323 endpoint terminals, a terminal and a gatekeeper, or a terminal and a gateway [6].

A brief description of the other protocols used by the H.323 is given as followed:

RAS is a transaction-oriented protocol between an H.323 endpoint and a gatekeeper. The endpoint uses RAS procedures to discover a gatekeeper, register or unregister with a gatekeeper, requesting call admission and bandwidth allocation, and call termination. A gatekeeper can enquire about the status of an endpoint using RAS.

H.225, which uses a subset of the ITU-T Q.931, is used for the call setup and teardown between two H.323 terminals.

H.245 is used for connection control, allowing two endpoints to negotiate media processing capabilities of the terminal, such as audio codec, for each media channel between them. It also determines master-slave relationships of the endpoints, and open and close logical channels between two endpoints [6].

Gatekeepers perform a number of important functions that preserve the integrity of the corporate data network. The first one is address translation from H.323 aliases for terminals and 
gateways to network addresses, as defined in the RAS specification. The second function is access control. The third function is bandwidth management. For instance, if a network manager has specified a threshold for the number of simultaneous conferences on the LAN, the Gatekeeper can refuse to make any more connections once the threshold is reached. The effect is to limit the total conferencing bandwidth; the remaining capacity is for email, file transfers, and other LAN activities. The fourth function is to manage a number of terminals, gateways, and MCUs as a single logical group known as the H.323 zone [7] [8].

\section{NEW SIGNALLING APPROACH IN AD HOC NETWORK}

\subsection{Motivation}

The topology of ad hoc networks is a known topology by its instability and its limitation in resources due to its nature running without infrastructure; and node mobility aggravates more the situation since every time the paths provided by the protocols for implemented routing should be updated which significantly increases the mechanism adopted to reach the destination in optimal time.

In order to be able to compensate for the degraded quality and service, it was necessary to think about a method of improving the quality of voice involving all the criteria limiters discussed above.

By taking advantage of the results of Chapter III where it was clear that the geographical position of the participating entities to a VoIP communication plays a vital role in terms of better quality of the obtained voice. For this, in case there is one mobile H.323 terminal, quality will be degraded unless certainly a solution is found, which will compensate for this degradation. This solution aims at minimizing 'the effort' provided by implementing three Gatekeepers instead of one; or wireless users calculate the distance between them; and the three; and Gatekeepers will decide which one will be chosen to connect the two terminals wishing to communicate via VoIP.

\subsection{Scenarios of VoIP within Ad-hoc simulated in Opnet Modeler}

Figure 1 illustrates an Ad hoc network model adopting mobile VoIP nodes and H.323 signalling entity (Gatekeeper) that supports all signalling operations for the entire topology.

Figure 2 illustrates the Ad hoc topology adopted in the case of the implementation of the three Gatekeepers instead of one or H.323 terminals desiring to initiate a VoIP call is addressed directly to the nearest Gatekeeper after calculating the distance to achieve each commissioned Gatekeeper.

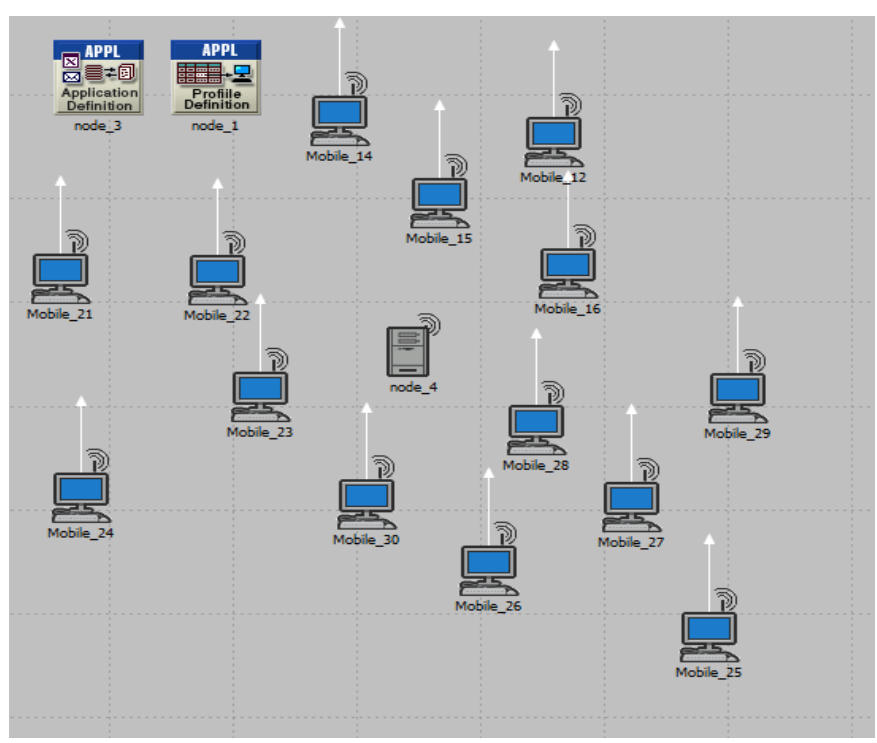

Figure 1: Ad Hoc Topology With Only One Gatekeeper

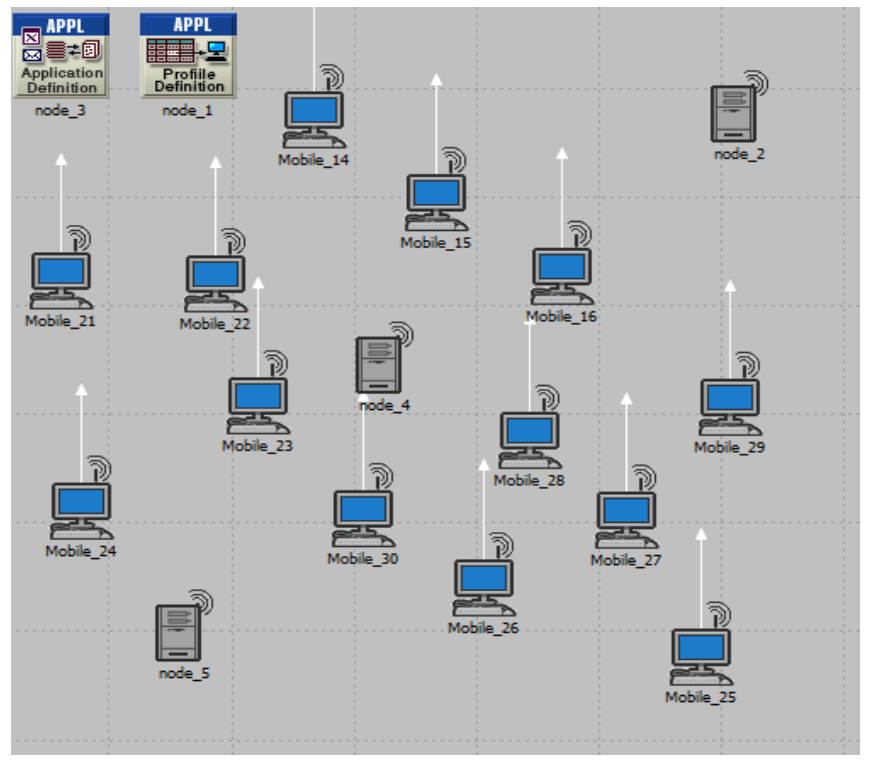

Figure 2: Ad Hoc Topology Suggested With Three Gatekeepers

\subsection{Integrated algorithm}

For the approach of the three Gatekeepers to be correctly operational, it is necessary to implement an algorithm that will allow the terminal to determine which of the three Gatekeepers they should address in order to reach the destination while keeping the highest quality possible.

As illustrated in Figure 3, a new algorithm has been implemented to allow terminals to determine which available Gatekeeper they should connect to.

The implemented algorithm involves that the terminal wishing initiate a call recovers its geographical coordinates $\mathrm{X}$ and $\mathrm{Y}$; using these coordinates it performs a calculation of distances D1, D2 and D3 that separate the Gatekeeper1 Gatekeeper2 Gatekeeper3 respectively which are considered to be fixed entities (fixed coordinates $\mathrm{X}$ and $\mathrm{Y}$ ). Distances are calculated according to the following finitary relation:

$$
\begin{aligned}
& D_{1}=\sqrt{ }\left(\left(x_{1}-X\right)^{2}+\left(y_{1}-Y\right)^{2}\right) \\
& D_{2}=\sqrt{ }\left(\left(x_{2}-X\right)^{2}+\left(y_{2}-Y\right)^{2}\right)
\end{aligned}
$$




$$
\mathrm{D}_{3}=\sqrt{ }\left(\left(\mathrm{x}_{3}-\mathrm{X}\right)^{2}+\left(\mathrm{y}_{3}-\mathrm{Y}\right)^{2}\right)
$$

with $(x 1, y 1),(x 2, y 2)$ and (x3, y3) being the known coordinates respectively to screen the three Gatekeepers 1,2 and 3 .

After making the necessary calculations, the terminal makes a comparison between the three calculated distances. So if D1 has the minimum value, the terminal must therefore address the Gatekeeper1. If instead D2 is the smallest distance then it must go to Gatekeeper2. Otherwise, it addresses the Gatekeeper3.

\subsection{Program coded in $\mathrm{C}$}

As it is mentioned in the previous sections, the Opnet Simulator offers the possibility to program new network entities and thus be able to test their feasibility and impact of integration to new environments.

The program below is the update of the h323_mgr_dynamically_find_assigned_gatekeeper () function implemented to discover the Gatekeeper, called later in the Header Block to run with hundreds of other functions in order to accomplish the task of H.323 signalling.

\subsection{Node Model:}

As illustrated in Figure 8, the newly created model Gatekeeper node contains a number of modules which are the basic building blocks of the model that offer all CPU features and can also buffer and manage the collection of data packets.

"Mac" and "Wireless LAN Mac" are used to connect to wired and wireless networks.

The blocks "Mac" and "Wireless LAN Mac" obviously contain sub-nodes called process model that illustrates the compilation of an algorithm translated into $\mathrm{C}$.

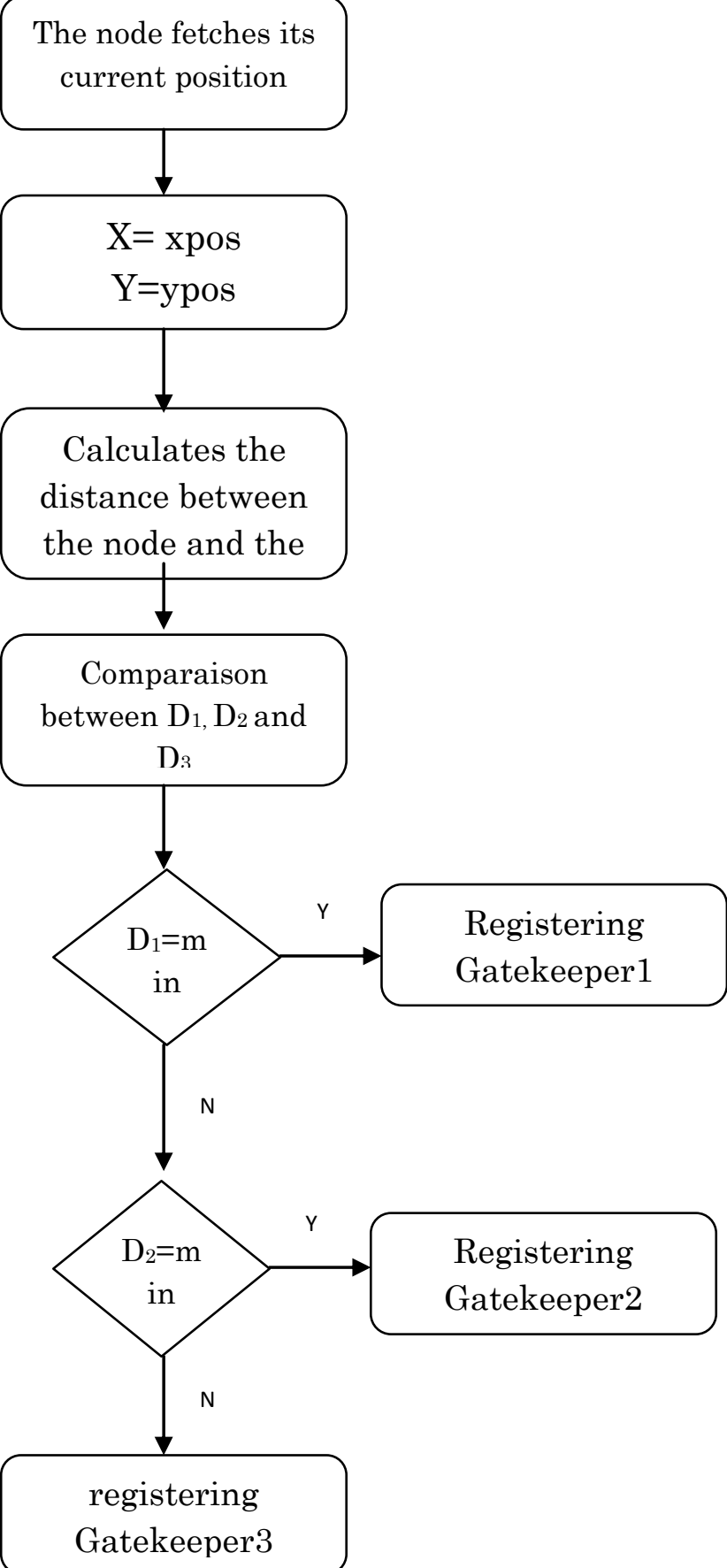

Figure 3: Suggested Improvement

\section{ASSESSMENT OF QOS \\ PARAMETERS OF THE SUGGESTED APPROACH}

This section contains the results obtained during the implementation of the new approach based on 3 Gatekeepers that are selected at once from the self-geographic location of the user.

\subsection{Jitter}

The variation of end-to-end delay between two consecutive packets is called jitter. Jitter less than $50 \mathrm{~ms}$ is acceptable for high quality VoIP calls. 
If the transmission delay varies too much in a VoIP call, the call quality deteriorates sharply. [7]

As shown in Figure 4, the new H.323 signalling approach could lead to a very important result in terms of jitter, which contributes to improving the quality of voice service.

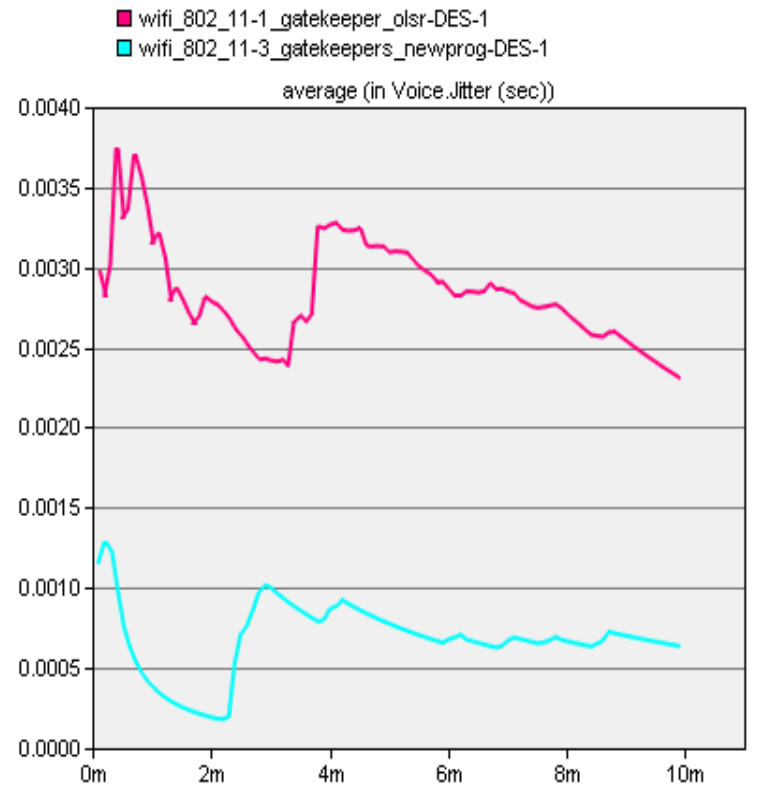

Figure4: JITTER

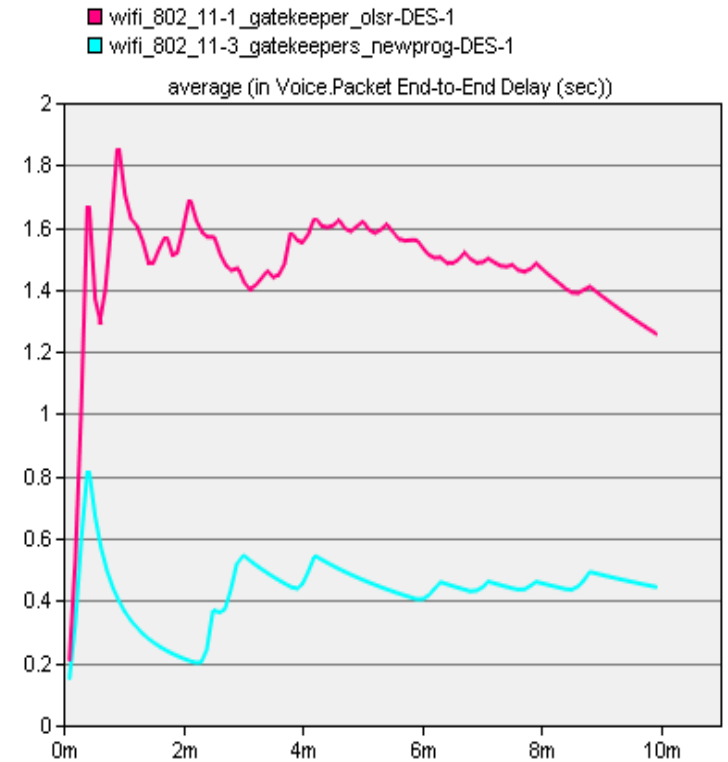

Figure 5: End To End Delay

\subsection{End-to-end delay}

The end to end delay consists of the delay of end system and network. The delay of the end system is taken for the delay of the encoding, decoding and buffering time [9].

Figure 5 also shows that in the case of calculating the delay end to end the approach of the three Gatekeepers was able to show high skills or it helped to gain a delay of approximately $1 \mathrm{~s}$.

\subsection{Packet loss rate (sent / received packets)}

As shown in both Figures 6 and 7, the packet loss rate is much higher in the case of single Gatekeeper; while in the case of three Gatekeepers, the loss rate is very optimal. This proves that the results obtained during the implementation of the new approach are very important and promote the suggested choice compared to other techniques for improving the quality of service.

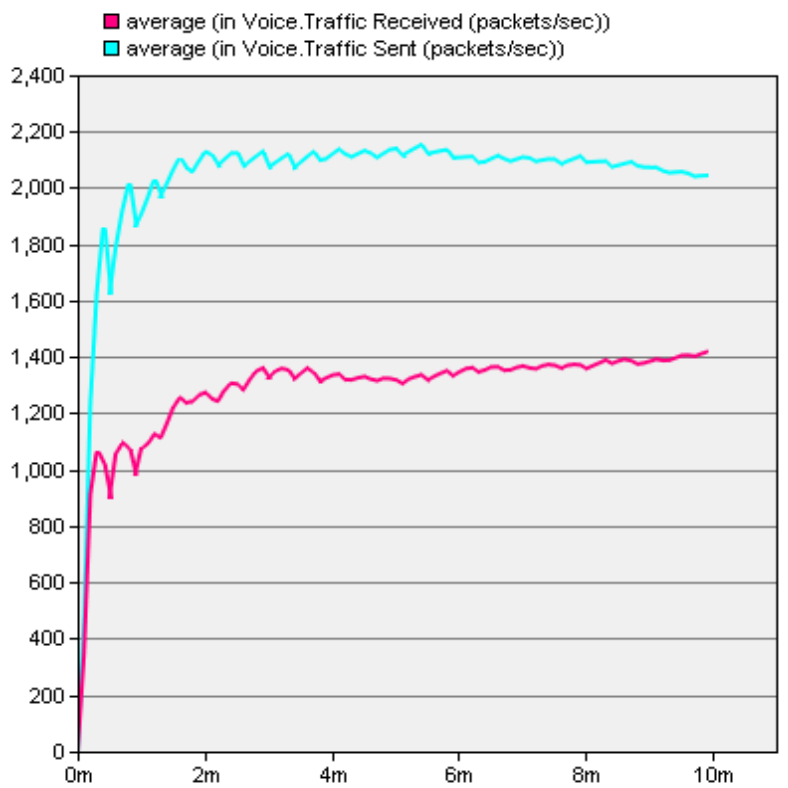

Figure 6: Received And Sent Traffic In The Case Of A Single Gatekeeper

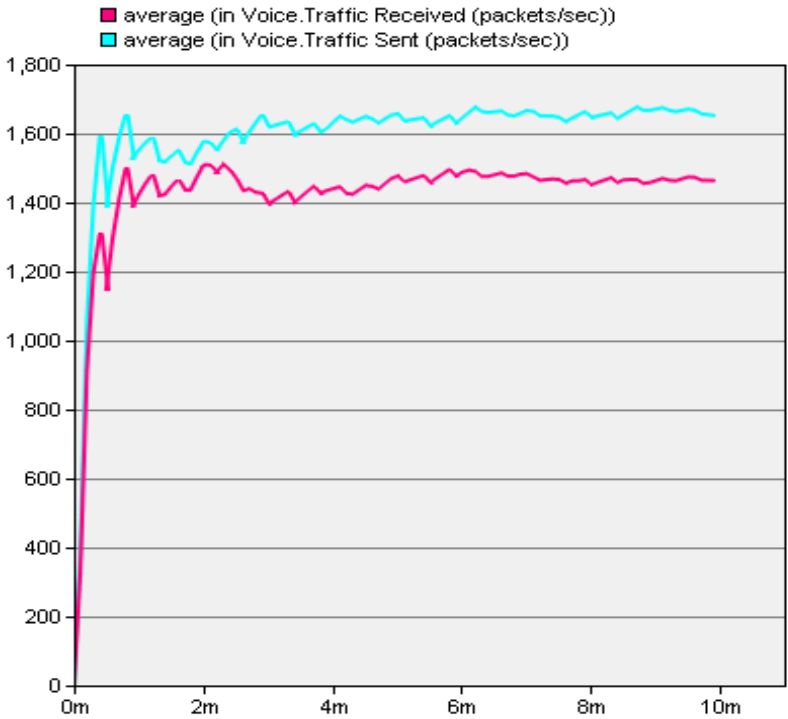

Figure 7: Received And Sent Traffic In The Case Of Thee Gatekeepers 


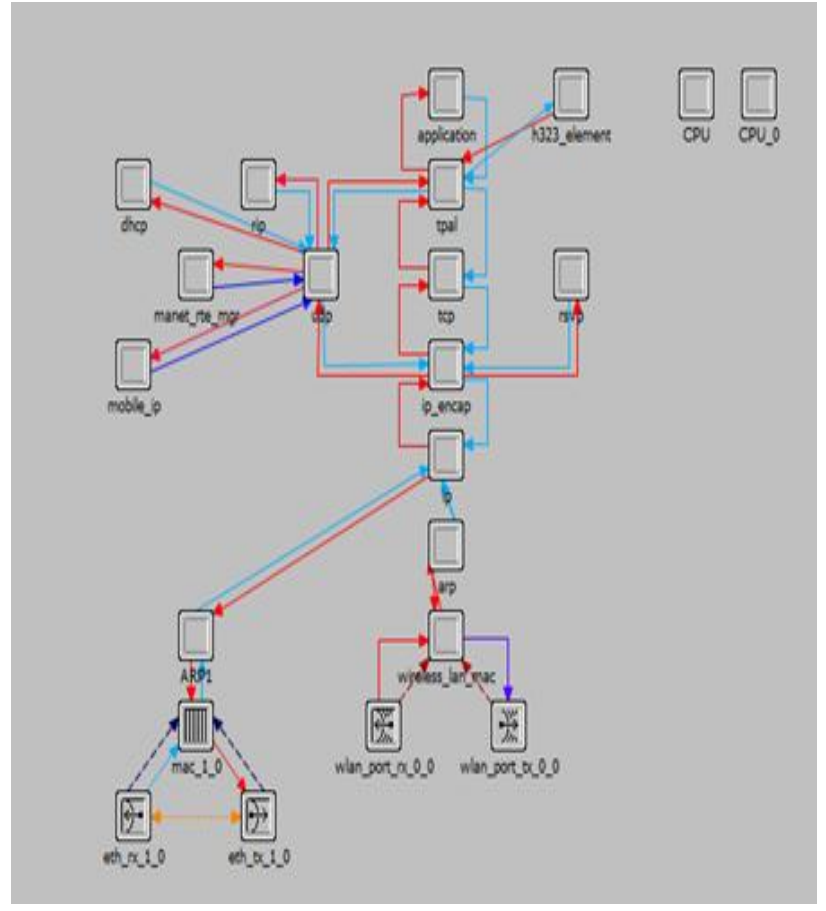

Figure 8: The Newly Created Node Model

\section{CONCLUSION}

In brief, this article is a summary of the implementation of a new H.323 signalling approach based on the implementation of three Gatekeepers in the same ad hoc network topology carrying VoIP where terminals / Call initiators get their contact information $\mathrm{X}$ and $\mathrm{Y}$ and automatically calculate the distances between the three available Gatekeepers; the minimum distance will be the basic criterion for deciding the chosen Gatekeeper.

This new approach could lead to important results in terms of service quality, in which all the basic criteria (jitter, end to end delay, packet loss rate) are considered favourable or very beneficial by comparing the two topologies (one / three Gatekeepers).

In perspective, it is planned to improve the new node created and presented under the third chapter and adapt it to integrate it into an ad hoc network, which will improve the quality of VoIP, be it based on SIP signalling or H.323 signalling.

\section{ACKNOWLEDGEMENTS}

This work falls within the scope of telecommunication projects. We would like to thank the Department of technology of the MESFCRST for financing our projects.

\section{CONFLICT OF INTEREST}

The authors declare that there is no conflict of interest regarding the publication of this manuscript.

\section{REFERENCES}

[1] Mobile ad-hoc network. Wikipedia. [Online] http://en.wikipedia.org/wiki/Mobile_ad-hoc_network. Dec. 2014

[2] ITU-T Recommendation G.114. "One way transmission time" 2003

[3] Mobile ad-hoc network. Wikipedia [Online]http://en.wikipedia.org/wiki/Mobile_adhoc_network. dec. 2014

[4] Maltz,David A. Johnson, David B. "Protocols for adaptive wireless and mobile computing". IEEE Personal Communications. 1996.

[5] "H.323 - Packet based Multimedia Communication Systems", ITU-T standard 09/99.

[6] Anoop Kumar K, Malhotra, T., "A multi-signaling protocol architecture for voice over IP terminal", INFOCOM 2004.

[7] Twenty-third Annual Joint Conferences of the IEEE Computer and Communications Societies, Volume 2, 7 11 March2004 Page(s):1191 - 1199 vol.2

[8] S. Rattal, A. Badri and M. Moughit. "Performance Analysis of Hybrid Codecs G.711 and G.729 over Signaling Protocols H.323 and SIP". International Journal of Computer Applications (IJCA) Vol.72 No.3: pages30-33, June 2013. Published by Foundation of Computer Science, New York, USA

[9] ITU-T Recommendation H.323, Packet-based multimedia communications systems, November 2000.

[10] Amit Chhabra Dr. Gurpal Singh "Performance Evaluation and Delay Modelling of VoIP Traffic over 802.11 Wireless Mesh Network" International Journal of Computer Applications (0975 - 8887) Volume 21- No.9, May 2011 